\title{
SOUTHERN METHODIST UNIVERSITY
}

\section{DTIC FILE COPY}

$$
A D-A 223280
$$


SADDLEPOINT APPROXIMATIONS IN CONDITIONAL INFERENCE

Suojin Wang

Southern Methodist University

SMU/DS/TR-239

3 


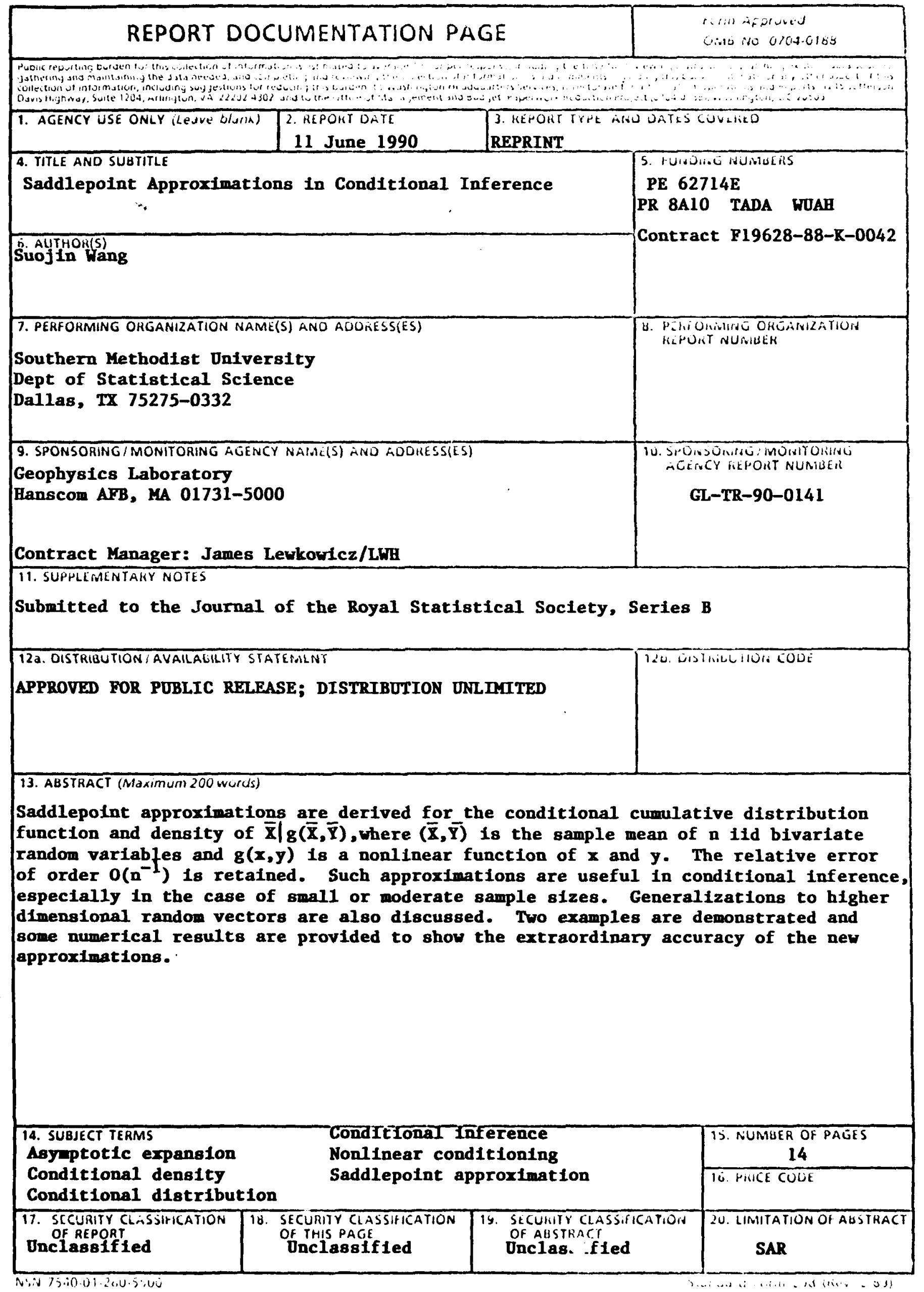




\title{
Saddlepoint Approximations in Conditional Inference
}

\author{
Suojin Wang \\ Department of Statistical Science \\ Southern Methodist University \\ Dallas, TX 75275
}

\section{SUMMARY}

Saddlepoint approximations are derived for the conditional cumulative distribution function and density of $\bar{X} \mid g(\bar{X}, \bar{Y})$, where $(\bar{X}, \bar{Y})$ is the sample mean of $n$ iid bivariate rasdom variablea and $g(x, y)$ is a nonlinear function of $x$ and $y$. The relative error of order $O\left(a^{-1}\right)$ is retained. Such approximations are useful in conditional inference, especially in the case of small or moderate sample sizes. Generalizations to higher dimensional random vectors are also discussed. Two examples are demonstrated and some numerical results are provided to show the extraordinary accuracy of the new approximations.

Key words: Asymptotic expansion; Conditional density; Conditional distribution; Conditional inference; Nonlinear conditioning; Saddlepoint approximation.

\section{INTRODUCTION}

Conditional inference plays an important role in statistical inference. The conditionality principle has been used to deal with various problems. Recent developments on this issue include important contributions by Barndorff-Nielsen (1980), Cox and Reid (1987), Fraser and Reid (1988), Diciccio, Fieid and Fraser (1990) and others. One major step in the procedure of conditional inference is to obtain the conditional distribution functions. As is often the case, the exact conditional distributions are difficult or impossible to obtain, and conventional approximations may often fail to work. For example, generaily it is hard to calculate the moments of the conditional distributions which are 
necessary quantities for the Edgeworth approximations. Furthermore, these approximations are often unsatisfactory for smsill or moderate sample sizes. -

On the other hand, it is well known that saddlepoint expansions lead to accurate approximations. even for small sample sizes. Among other papers, Barndorff-Nieisen and Cox (1979) and Reid (1988) have discussed the importance and usefulness of the saddlepoint approximations in statistics. Davison and Hinkley (1988) apply the saddlepoint method (see Daniels (1987)) to resampling problems. Using the saddlepoint technique twice, Skovgaard (1987) bas derived an accurate approximation to the conditional distribution of a sample mean given a $p-1$ dimensional linear function of a $p$ dimensional variable which has been applied by Davison (1988) and Davison and Hinkley (1988) to generalized linear models and resampling analysis. Wang (1990b) derives saddlepoint formulas for bivariate distribution functions. But it is seen that most applications are limited to the sample mean and other simple linear statistics due to the strong requirements of the saddlepoint technique on the moment generating function of the statistics under consideration.

However, consideration of more general statistics is often desired. In particular, conditional inference often requires a distribution function conditioning on nonlinear functions of a sample mean of multivariate random variables. Recent work on saddlepoint expansions includes expansions for some specific nonlinear statistics by Srivastava and Yau (1989) and Wang (1990c).

Fin this paper we derive accurate saddlepoint expansions for the case of nonlinear conditioning. The results include Skovgaard's (1987) method as a special case when the distribution is continuous, but have much broader applications. Sections 2 and 3 expand saddlepoint formulas for the conditional density and conditional distribution function, respectively. Two examples are considered in Section 4 to illustrate the use of the new results. Extraordinary accuracy is also shown numerically.

\section{SADDLEPOINT EXPANSIONS FOR THE CONDITIONAL DE.VSITY}

We consider first the bivariate case, and generalize results in the $p(>2)$ dimensional case at the end of Section 3. Assume that $\left(X_{1}, Y_{1}\right), \ldots,\left(X_{n}, Y_{n}\right)$ are independent identically distributed 
bivariate continuous random variables with the cumulant generating function $K(u, v)$ existing in a neighborbood of $(0,0)$. Let

$$
T=c_{1} \bar{X}+c_{2} \bar{Y}
$$

and $\mathrm{Z}=g(\overline{\mathrm{X}}, \overline{\mathrm{Y}})$ be a bivariate function on the sample space, where $c_{1}$ and $c_{2}$ are given constants, $\overline{\mathrm{X}}$ and $\bar{Y}$ be the sample means of $X_{i}$ 's and $Y_{i}$ 's, respectively. We wish to expand the conditional distribution $B_{n}\left(t_{0} \mid z\right)=\operatorname{Pr}\left(T<t_{0} \mid Z=z\right)$ and the corresponding density. Without loss of generality, let $T=\bar{X}$ and ascume that the transformation from $(\bar{X}, \bar{Y})$ to $(T, Z)$ is one to one. Then the inverse transform can be written as $(\bar{X}, \bar{Y})=(T, q(T, Z))$ for some function $q$. When the transform is not one to one, the domain should be partitioned so that in each portion the transform is one to one.

In this section we consider the conditional density. It is well known that the density $\mathrm{P}_{n}(x, y)$ of $(\bar{X}, \bar{Y})$ has the following saddlepoint approximation (equation (1) of Reid (1988)):

$$
\operatorname{Pn}(x, y)=P_{s}(x, y)\left\{1+O\left(n^{-1}\right)\right\}
$$

where

$$
P_{s}(x, y)=\frac{n}{2 \pi\{D(\hat{u}, \hat{u})\}^{2 / 2}} \exp \{n[K(\hat{u}, \hat{u})-\hat{u} x-\hat{u} y]\}
$$

$D(u, v)=\left.\left\{\ddot{K}_{u u} \ddot{K}_{v v}-\left(\ddot{K}_{u v}\right)^{2}\right\}\right|_{(u, v)}=|\ddot{K}(u, v)|$, the determinant of the $2 \times 2$ matrix of the second partial derivatives of $\mathrm{K}$, and $(\hat{u}, \hat{v})$ is the saddlepoint defined by

$$
\left\{\begin{array}{l}
\frac{\partial}{\partial u} K(\hat{\mathbf{u}}, \hat{v})=x \\
\frac{\partial}{\partial \mathbf{v}} K(\hat{\mathbf{u}}, \hat{\mathbf{v}})=y
\end{array}\right.
$$

Here we adapt Skovgaard's notations for partial derivatives, e.g., $\ddot{\mathrm{K}}_{\mathrm{uu}}$ is the second partial derivative of $\mathrm{K}$ with respect to $\mathrm{u}$, etc. The relative error in (1) is of order $\mathrm{n}^{-1}$ uniformly over compact sets. In this paper we will use " $\simeq$ " to denote an approximation having such an error. Letting $(x, y)=(t, q(t$. z)) and still using ( $(\hat{u}, \hat{v})$ to denote the corresponding solution in (3), it is easily seen from (1) that the density $h_{n}(t, z)$ of $(T, Z)$ can be expressed as

$$
h_{n}(t, z)=p_{n}(t, q(t, z))|J| \simeq h_{s}(t, z),
$$


where

$$
h_{s}(t, z)=\frac{n|J|}{2 \pi\{D(\hat{u}, \hat{v})\}^{1 / 2}} \exp \{n[K(\hat{u}, \hat{v})-\dot{u} t-\hat{u} q(t, z)]\}
$$

and $J=\dot{q}_{z}(t, z)=\left\{\dot{q}_{y}(x, y)\right\}^{-1}$ is the Jacobian factor. To approximate the conditional density $h_{n}(t \mid z)$ of $T \mid(Z=z)$, it is sufficient to obtain an approximation to the marginal density $h_{n}(z)$ for $Z$. By (4),

$$
h_{n}(z)=\int_{-\infty}^{\infty} h_{n}(t, z) d t \simeq \int_{-\infty}^{\infty} h_{s}(t, z) d t
$$

Let

$$
L(t)=K(\hat{u}, \hat{v})-\hat{u} t-\hat{v} q(t, z),
$$

where $z$ is fixed so that $\hat{\mathrm{u}}$ and $\hat{\mathrm{v}}$ depend on $t$ only. Then by (3),

$$
L^{\prime}(t)=-\hat{u}-\hat{v} \dot{q}_{t}(t, z)
$$

and

$$
I^{\prime \prime}(t)=\left\{-\ddot{K}_{v v}(\hat{u}, \hat{v})+2 \dot{q}_{t}(t, z) \ddot{K}_{u v}(\hat{u}, \hat{v})-\left[\dot{q}_{t}(t, z)\right]^{2} \ddot{K}_{u u}(\hat{u}, \hat{v})\right\} / D(\hat{u}, \hat{v})-\hat{v} \ddot{q}_{t t}(t, z),
$$

since by taking derivative with respect to $t$ of both sides of $(3)$ when $(x, y)=(t, q(t, z))$ we have

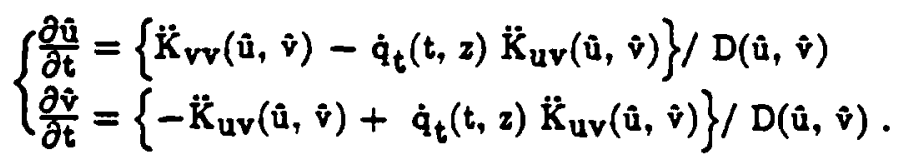

We now assume that there exists a unique solution, $t_{1}$, to

$$
L^{\prime}(t)=0
$$

and $L^{\prime \prime}\left(t_{1}\right)<0$. Following the technique by Bleistein (1966), we transform $t$ to w such that

$$
\left(w-w_{1}\right)^{2} / 2=L\left(t_{1}\right)-L(t),
$$

where $w_{1}=\operatorname{sgn}\left(t_{1}\right)\left\{2\left[L\left(t_{1}\right)-L(0)\right]\right\}^{1 / 2}$ and $\operatorname{sgn}\left(w-w_{1}\right)=\operatorname{sgn}\left(t-t_{1}\right)$. Note that $w=0$ and $w=w_{1}$ correspond to $t=0$ and $t=t_{1}$, respectively. From (4), (5) and (10), it is seen that 


$$
\begin{aligned}
h_{n}(z) & \simeq \frac{n}{2 \pi} \exp \left\{n L\left(t_{1}\right)\right\} \int_{-\infty}^{\infty} \exp \left\{-n\left(\nabla-\nabla_{1}\right)^{2} / 2\right\} \frac{|J|}{\{D(\hat{u}, \hat{v})\}^{1 / 2}} \frac{d t}{d w} d w \\
& \left.\simeq\left\{\frac{n}{2 \pi}\right\}^{1 / 2} \exp \left\{n L\left(t_{1}\right)\right\}\left\{\frac{|J|}{\{D(\dot{u}, \hat{v})\}^{1 / 2}} \frac{d t}{d w}\right\}\right|_{w=w_{1}} .
\end{aligned}
$$

This last approximation is obtained by appiying Watson's lemma (Jeffreys and Jeffreys (1962)). But by differentiating both sides of $(10)$, we have

$$
\left.\frac{d t}{d w}\right|_{w=w_{1}}=\lim _{t \rightarrow t_{1}}-\frac{w-w_{1}}{L^{\prime}(t)}=\left\{-L^{\prime \prime}\left(t_{1}\right)\right\}^{-1 / 2}
$$

Thus,

$$
h_{n}(z) \simeq\left\{-\frac{n}{2 \pi L^{\prime \prime}\left(t_{1}\right) D\left(\dot{u}_{1}, \dot{v}_{1}\right)}\right\}^{1 / 2}\left|J_{1}\right| \exp \left\{n L\left(t_{1}\right)\right\}
$$

where $\left(\hat{u}_{1}, \hat{v}_{1}\right)$ is the solution to $(3)$ when $(x, y)=\left(t_{1}, q\left(t_{1}, z\right)\right)$, and $J_{1}=\dot{q}_{z}\left(t_{1}, z\right)$ is the Jacobian factor at $t=t_{1}$. Noticing that the conditional density is given by

$$
h_{n}(t \mid z)=\frac{h_{n}(t, z)}{h_{n}(z)}
$$

from (4), (6) and (12) we have reached the following result.

Theorem 1. Let $T=\bar{X}, Z=g(\bar{X}, \bar{Y})$. Assume that the general regularity conditions described at the beginning of this section hold and that the solution $t_{1}$ in (9) exists. Denote the inverse transform by $(X, \bar{Y})=(T, q(T, Z))$. Then the conditional density of $T$ given $Z=2$ has the saddlepoint expansion

$$
b_{n}(t \mid z) \simeq\left\{\frac{-n L^{\prime \prime}\left(t_{1}\right) D\left(\hat{u}_{1}, \dot{v}_{2}\right)}{2 \pi D(\dot{u}, \dot{v})}\right\}^{1 / 2}\left|\frac{J}{J_{1}}\right| \exp \left\{n\left[L(t)-L\left(t_{1}\right)\right]\right\}
$$

where $(\hat{u}, \hat{v})$ and $\left(\dot{u}_{1}, \hat{v}_{1}\right)$ are the solutions to $(3)$ when $(x, y)=(t, q(t, z))$ and $\left(t_{1}, q\left(t_{1}, z\right)\right)$, respectively, and $J$ and $J_{1}$ are the Jacobian factor at $t$ and $t_{1}$, respectively. 
As is seen in the next section, the technique used to derive (12) can be modified for the purpose of approximating the conditional distribution function. Expanaion (12) is useful in the derivations.

\section{SADDLEPOINT EXPANSION FOR THE CONDITIONAL DISTRIBUTION}

We begin with the following lemma which will be needed in this section.

Lemma 1. Assume that $\alpha(x)$ is an analytic function. Then

$$
\left(\frac{n}{2 \pi}\right)^{1 / 2} \int_{-\infty}^{y} \alpha(x) \exp \left\{-n x^{2} / 2\right\} d x \simeq \alpha(0) \Phi(\sqrt{n} y)-\frac{\alpha(y)-\alpha(0)}{\sqrt{n} y} \phi(\sqrt{n} y),
$$

where $\phi$ and $\Phi$ are the standard normal density and cumulative distribution function, respectively. When $y=0$, the expansion is $\alpha(0) / 2-\alpha^{\prime}(0) /(2 \pi \mathrm{n})^{1 / 2}$.

This lemma is a special case of Temme (1982); see Temme (1982) for the proof.

By (4) and (6), the conditional distribution function is

$$
\begin{aligned}
& H_{n}\left(t_{g} \mid z\right)=\frac{1}{h_{n}(z)} \int_{-\infty}^{t_{0}} b_{n}(t, z) d t \\
& \simeq \frac{n}{2 \pi h_{n}(z)} \int_{-\infty}^{t_{0}} \frac{|J|}{\{D(\hat{u}, \hat{v})\}^{1 / 2}} \exp \{n L(t)\} d t .
\end{aligned}
$$

Using the same transformation as in (10) and denoting by $\boldsymbol{w}_{0}$ the transformed value of $t$ evaluated at $t_{0}$, we have

$$
H_{n}\left(t_{0} \mid z\right) \simeq \frac{n \exp \left\{n L\left(t_{i}\right)\right\}}{2 \pi h_{n}(z)} \int_{-\infty}^{\Phi_{0}} c(w) \exp \left\{-n\left(w-w_{l}\right)^{2} / 2\right\} d w
$$

where $c(w)=|J| \frac{d t}{d w} /\{D(\hat{u}, \dot{v})\}^{1 / 2}$. Therefore by Lemma 1 and formulas (11) and (12), it is easily seen that 


$$
\begin{aligned}
B_{n}\left(t_{0} \mid z\right) & \simeq\left(\frac{n}{2 \pi}\right)^{1 / 2} \frac{\exp \left\{n L\left(t_{1}\right)\right\}}{h_{n}(z)}\left\{c\left(w_{1}\right) \Phi\left(a_{0}\right)-\frac{c\left(w_{0}\right)-c\left(w_{0}\right)}{a_{n}} \phi\left(a_{n}\right)\right\} \\
& \simeq \Phi\left(a_{0}\right)+\left\{\frac{1}{a_{0}}-\frac{1}{b_{0}}\right\} \phi\left(a_{0}\right),
\end{aligned}
$$

where

$$
\begin{aligned}
& a_{0}=\sqrt{\mathbf{n}}\left(w_{0}-\varpi_{1}\right)=\operatorname{sgn}\left(t_{0}-t_{1}\right)\left\{2 n\left[L\left(t_{1}\right)-L\left(t_{0}\right)\right]\right\}^{1 / 2}, \\
& b_{0}=\frac{a_{0} c\left(\varpi_{1}\right)}{c\left(w_{0}\right)}=-L^{\prime}\left(t_{0}\right)\left|\frac{J_{1}}{J_{0}}\right|\left\{-\frac{n D\left(\dot{u}_{0}, \dot{v}_{0}\right)}{L^{\prime \prime}\left(t_{1}\right) D\left(\dot{u}_{1}, \hat{v}_{1}\right)}\right\}^{1 / 2},
\end{aligned}
$$

and $J_{0}$ and $J_{1}$ are the Jacobian factor at $t_{0}$ and $t_{1}$, respectively. We summarize the above results in the following theorem.

Theorem 2. Under the conditions in Theorem 1, the conditional distribution function $B_{n}\left(t_{g} \mid z\right)=\operatorname{Pr}(T$ $<t_{0} \mid Z=2$ ) has the saddiepuint expansion (17). When $t_{0}=t_{1}$, the expansion can be obtained by taking the limit of (17) as $t_{0} \rightarrow t_{1}$.

Notice that formula (17) has the same form as Lugannani and Rice's (1980) formula, as well as those of Skovgaard (1987) and Wang (1990c). Note also that because of the special structure of the saidlepoint technique the expansions (4), (12), (14) and (17) with relative exror of order $n^{-1}$ are sufficiently accurate for moet applications. Higher order expansions could be obtained by referring to Temme (1982), although it is algebraically complicated.

It is straightforward to generalize expansions (14) and (1i) to the $p(>2)$ dimensional case with an analog of Skovgaard (198i). Let $\left(X_{i}, Y_{i}\right), i=1, \ldots, a$, be ïd continuous $p$ dimensional random vectors. Assume that $(T, Z)=(\bar{X}, g(\bar{X}, \bar{Y}))$ is a one to one transform. where $Z=g(\bar{X}, \bar{Y})$ is a $p-1$ dimensional vector, and that expansions for $B_{n}\left(t_{0} \mid z\right)=\operatorname{Pr}\left(T<t_{0} \mid Z=z\right)$ and the density $h_{n}\left(t_{0} \mid z\right)$ are desired. Then the inverse transform $(\bar{X}, \bar{Y})=(T, q(T, Z))$ exists and the variable $v$ in the cumulant generating function $K(u, v)$ of $(X, \bar{Y})$ is $p-1$ dimensional. Analogously to (6), let 


$$
L(t)=K(\dot{u}, \hat{v})-\dot{u} t-\dot{v}^{T} q(t, z),
$$

where $v^{\mathcal{I}}$ is the transpose of $\dot{v}$, and let $J=\frac{\partial(x, y)}{\partial(t, z)}$ be the Jacobian factor and $D(u, v)$ be the determinant of the $p \times p$ matrix of the second partial derivatives of $K(u, v)$. Foilowing ibe same procedures, it is easily seen that (14) and (17) also hoid for the general p dimensional case.

\section{EXAMPLES}

In this section we consider two applications. The first one is about the conditional density and conditional distribution of $\bar{X} \mid \dot{\sigma}$, where $\dot{\sigma}^{2}=\frac{1}{n-1} \Sigma\left(X_{i}-\bar{X}\right)^{2}$. It is equivalent to consider $\bar{X} \mid\left(\frac{1}{\mathrm{~L}} \Sigma X_{\mathrm{i}}^{2}-(\overline{\mathrm{X}})^{2}\right)$. Let $\mathrm{T}=\overline{\mathrm{X}}, \overline{\mathrm{Y}}=\frac{1}{2} \Sigma \mathrm{X}_{\mathrm{i}}^{2}$ and $\mathrm{Z}=\mathrm{g}(\overline{\mathrm{X}}, \overline{\mathrm{Y}})=\overline{\mathrm{Y}}-(\overline{\mathrm{X}})^{2}$. Then the probiem of approximating $\operatorname{Bn}_{n}\left(t_{0} \mid z\right)=\operatorname{Pr}\left(T<t_{0} \mid Z=z\right)$ and its density $h_{n}(t \mid z)$ can be handled weil by the new results developed in this paper, provided that the general conditions in Section 2 are satisfied. In particular, when $X_{i}$ 's are normally distributed, say from $\mathrm{N}(0,1)$, it is easily calculated that the saddlepoint expansions (14) and (17) are $h_{s}(t \mid 2)=\sqrt{a} \phi(\sqrt{a} t)$ and $H_{s}\left(t_{0} \mid z\right)=\Phi\left(\sqrt{n} t_{0}\right)$, independent of z. That is, the approximations become exact in this case.

We shall discuss the second example in a more detailed manner. In an interesting paper, Hinkley (1977) posed the following problem. Let $W_{1}, \ldots, W_{n}$ be independently $\mathrm{N}\left(\mu, c^{2} \mu^{2}\right)$, where $c$ is a known constant and $\mu>0$ is the parameter of interest. For simplicity, let $c=1$. We may taike as an ancillary statistic

$$
z=\bar{W} /\left\{\frac{1}{\mathbf{D}} \Sigma w_{i}^{2}\right\}^{1 / 2},
$$

as pointed out by Binikley. By the conditionality principle (Cox and Hinkley (19i4), Sec. 2.2), inference about $\mu$ is based on the conditional distribution of $T=\frac{1}{\mathrm{a}} \Sigma \mathrm{W}_{\mathrm{i}}^{2}$ given the observed value of $Z$. A major step in the problem is to obtain $H_{n}\left(t_{g} \mid z\right)$ and $h_{n}\left(t_{g} \mid z\right)$ or sufficiently accurate approximations to them. Expansions (14) and (1i) serve this purpose. To illustrate, let the true vaiue $\beta=1$ and let $X_{i}=W_{i}^{2}$ and $Y_{i}=W_{i}$. Thus by $(18), z=g(x, y)=y / \sqrt{x}$ and $y=q(t . z)=\sqrt{t} z$. It is easily obcained that the cumulant generating function of $\left(X_{i}, Y_{i}\right)$ is

$$
K(u, v)=u+v+\frac{(2 u+v)^{2}}{2(1-2 u)}-\frac{1}{2} \log (1-2 u) \text {. }
$$


For $(x, y)=(t, \sqrt{t} z)$, the solution to $(3)$ is

$$
(\hat{u}, \dot{v})=\left(\frac{1}{2}-\frac{1}{2\left(1-z^{2}\right) t}, \frac{\sqrt{t} z}{\left(1-z^{2}\right) t}-1\right) .
$$

From (6), some algebra leads to

$$
L(t)=z \sqrt{t}-\frac{t}{2}+\frac{1}{2} \log t+\frac{1}{2} \log \left(1-z^{2}\right) .
$$

Note that $|z|<1$. Hence the solution $t_{1}$ to $L^{\prime}(t)=0$ is given by

$$
t_{1}=\left(z^{2}+2+z \sqrt{2^{2}+4}\right) / 2 \text {, }
$$

and the condition

$$
L^{\prime \prime}(t)=-2 t^{-3 / 2} / 4-t^{-2} / 2<0
$$

holds for all $\mathrm{t}$ in its domain $(0, \infty)$. Moreover, one can obtain that

$$
D(\hat{u}, \hat{v})=2\left\{\left(1-z^{2}\right) t\right\}^{3} \text { and }|J|=\sqrt{t} \text {. }
$$

Therefore, expansion (14) reduces to

$$
h_{n}(t \mid z) \simeq c_{n} t^{n / 2-1} \exp \left\{-n(\sqrt{t}-2)^{2} / 2\right\}
$$

where

$$
c_{n}=\frac{1}{2}\left\{\frac{n}{7}\left(\frac{2 \sqrt{t_{1}}}{2}+1\right)\right\}^{1 / 2} t_{1}^{-n / 2} \exp \left\{n\left(\sqrt{t_{1}}-2\right)^{2} / 2\right\}
$$

Since the exact density $h_{n}(t \mid z)$ is proportional to $t^{n / 2-1} \exp \left\{-n(\sqrt{t}-z)^{2} / 2\right\}$ (see Binkley (1977)), (20) is viewed as "exact" if the renormalization is allowed. Using the above calculations, expansion (17) for $B_{n}\left(t_{0} \mid 2\right)$ is easily computed with

$$
\begin{aligned}
& a_{0}=\operatorname{sgn}\left(t_{0}-t_{1}\right)\left\{n\left[\left(\sqrt{t_{0}}-\sqrt{t_{1}}\right)\left(\sqrt{t_{0}}+\sqrt{t_{1}}-2 z\right)+\log \left(t_{1} / t_{0}\right)\right]\right\}^{1 / 2}, \\
& b_{0}=\left(t_{0}-2 \sqrt{t_{0}}-1\right)\left\{n /\left(z \sqrt{t_{1}}+2\right)\right\}^{1 / 2} .
\end{aligned}
$$


Table 1 compares the saddlepoint approximation (17) with the true conditional distribution function for $z=0.5$ and $n=5,10$. The extraordinary accuracy of the approximation is evident uniformly over the domain of the statistic. Note that calculations show that the relative error is not affected by different values of $z$, i.e., the numerical accuracy of the expansion (17) is stabie for all $|z|$ $<1$.

\section{CONCLUDING REMARKS}

In this paper we have derived genuine saddlepoint expansions for conditional densities and distributions with nonlinear conditioning. The resulting fomulas are highly accurate even for small sample sizes and are easy to use. Formula (17) reduces to Skovgaard's (1987) formula when the conditioning variable $Z=g(\bar{X}, \bar{Y})$ is a linear function of $\bar{X}$ and $\bar{Y}$.

Resampling methods such as bootstrap are very useful in conditional inference (see, for example, Hinkley (1988)). However, computational difficulty is generally a major obstacle in such approaches, evidently more so than in classical parametric problems. Besides their direct use in parametric applications, the new approximations provide a poesible remedy to overcome the difficulties in many such cases. Further investigations are needed to draw a solid conclusion on this issue. We have assumed continuity of the statistics in our derivations while those in the resampling schemes are discrete, although they become smooth as the sample size increases. There is now an interesting question as to whether the formulas derived here or their modified versions are valid for such purposes.

A detailed examination of this question is beyond the scope of this paper. Recent work by Wang (1990a) on the validity of saddlepoint expansions in the problem of bootstrapping a sample mean is relevant. 


\section{ACKNOWLEDGEMENT}

The author wishes to thank Professor D.V. Hinkley for initiaily raising the problem and Professor H.L. Gray for his encouragement on this research. It was partially supported by DARPA/AFGL Contract No. F19628-88-K-0042.

\section{REFERENCES}

Barndorff-Nielsen, O. (1980). Conditional resolutions. Biometrika, 67, 293-310.

Barndorff-Nielsen, O. and Cox, D.R. (1979). Edgeworth and saddlepoint approximations with staxistical applications (with discussion). J. R. Statist. Soc. B, 41, 279-312.

Cox, D.R. and Hinkley, D.V. (1974). Theoretical Stetistics. London: Chapman and Hall.

Cox, D.R. and Reid, N. (1987). Parameter orthogonality and approximate conditional inference (with discussion). J. R. Statist. Soc. B, 49, 1-39.

Daniels, H.E. (1987). Tail probability approximations. Int Statist Rev. 55, 37-48.

Davison, A.C. (1988). Approximate conditional inference in generalized linear models. J. R. Statist. Soc. B, 50, 445-461.

Davison, A.C. and Hinkley, D.V. (1988). Saddlepoint approximations in resampling methods. Biometrika 75, 41i-431.

Diciccio, T.J., Field, C.A. and Fraser, D.A.S. (1990). Approximations of marginal tail probabilities and inference for scalar parameters. Biometrika, 77, 77-95.

Fraser, D.A.S. and Reid, N. (1988). On conditional inference for a real parameter: A differential approach on the sample space. Biometrika, 75, 251-264.

Hinkley, D.V. (19Ti). Conditional inference about a normal mean with known coefficient of variation. Biometrike, 64, 105-108.

Hinkley, D.V. (1988). Bootstrap methods (with discussion). J.R. Statist. Soc. B, 50, 321-337.

Jeffreys. H. and Jeffreys. B.S. (1962). Methods of Mathematical Physics, 3rd ed. London and Vew York: Cambridge University Preas. 
Lugannani, R. and Rice, S. (1980). Saddlepoint approximation for the distribution of the sum of independent random variables. Adv. Appl Prob., 12, 475-490.

Reid, N. (1988). Saddlepoint methods and statistical inference (with discussion). Statist. Sci.. 3, 213238.

Skovgaard, I.M. (1987). Saddlepoint expansions for conditional distributions. J. AppL Prob., 24, 875887.

Srivastava, M.S. and Yau, W.K. (1989). Tail probability approximations of a general statistic. Unpublished manuscript.

Temme, N.M. (1982). The uniform asymptotic expansion of a clase of integrals related to cumulative distribution functions. SIAM J. Math. Anch, 13, 239-251.

Wang, S. (1990a). Saddlepoint approximations in resampling analysis. Ann. Inst. Statist. Math, 42, to appear.

Wang, S. (1990b). Saddlepoint approximations for bivariate distributions. J. Appl. Prob., 27, to appear.

Wang, S. (1990c). Saddlepoint approximations for nonlinear statistics. Ceradian J. Statist, 18, to appear. 
Table 1. Saddlepoint approximation (17) to the conditional distribution $B_{n}\left(t_{0} \mid z\right)$ in the second example in Section $4 ; 2=0.5$.

\begin{tabular}{|c|c|c|c|c|c|}
\hline \multicolumn{3}{|c|}{$n=5$} & \multicolumn{3}{|c|}{$a=10$} \\
\hline$t_{0}$ & saddlepoint & true & $t_{0}$ & saddlepoint & true \\
\hline 0.03 & .00004 & .00004 & 0.15 & .00002 & .00002 \\
\hline 0.05 & .00016 & .00016 & 0.20 & .00011 & .00011 \\
\hline 0.10 & .00103 & .00102 & 0.40 & .00351 & .00351 \\
\hline 0.30 & .01803 & .01803 & 0.60 & .02220 & .02223 \\
\hline 0.50 & .06295 & .06306 & 0.80 & .07111 & .07119 \\
\hline 0.70 & .13418 & .13446 & 1.00 & .15644 & .15660 \\
\hline 1.00 & .27298 & .27354 & 1.20 & .27179 & .27202 \\
\hline 1.40 & .47269 & .47348 & 1.50 & .46870 & .46900 \\
\hline 1.80 & .64674 & .64754 & 1.80 & .65067 & .65097 \\
\hline 2.20 & .77748 & .77815 & 2.10 & .79010 & .79034 \\
\hline 2.80 & .89815 & $898 \bar{i} \bar{T}$ & 2.40 & .88318 & .88335 \\
\hline 3.30 & .95012 & .95036 & 2.80 & .95160 & .95169 \\
\hline 4.00 & .98293 & .98303 & 3.30 & .98588 & .98591 \\
\hline 5.50 & .99860 & .99861 & 4.20 & .99884 & .99884 \\
\hline 6.50 & .99976 & .99977 & 4.70 & .99974 & .99974 \\
\hline 7.50 & 99996 & 99996 & 5.40 & T & 99997 \\
\hline
\end{tabular}

